\title{
Rezension zu Kauffeld \& Othmer (Hrsg.) (2019). Handbuch Innovative Lehre
}

\author{
Wiesbaden: Springer. 470 Seiten. 59,99€. ISBN 978-3-658-22796-8
}

\author{
Niclas Schaper ${ }^{1}$ \\ Online publiziert: 31. Januar 2020 \\ (c) Der/die Autor(en) 2020
}

Das Thema Digitalisierung wandelt nicht nur die Techniklandschaft, auch die Kultur in Organisationen verändert sich. „Neue Wissensformen gehen einher mit neuen Möglichkeiten des Lehrens und Lernens und werfen Fragen zur Aktualität vieler traditioneller und als selbstverständlich erachteter Lehrformen auf, die nicht zuletzt unser Selbstverständnis als Lehrende und die Hochschule als Ganzes irritieren können. Für jeden und jede Einzelne stellen sich viele neue, teils sehr konkrete, aber ebenso abstrakte Fragen [...]“ - so formulieren es die Herausgeber.

Noch vor der Beantwortung dieser konkreten Fragen wird zu Beginn des Buches am Beispiel der TU Braunschweig erläutert, wie innovative Lehr- und Lehrideen nachhaltig an der Hochschule verankert werden können und diese die Lehr-Lernkultur verändern können. Dies geschieht, wie insgesamt in diesem Buch, auf eine sehr anschauliche Art und Weise. Es gibt eine Vielzahl von Hinweisen, die praxisorientiert vor dem Hintergrund der aktuellen Literatur zu Veränderungsprozessen reflektiert werden. Wie kann es gelingen die Universität in Bewegung zu bringen? Wie können einzelne Innovationsprojekte die Universität verändern? Wie können Innovationsprojekte über Transferprojekte in die Breite getragen werden? Welche Bedeutung können Akademische Fachzirkel für Neuerung haben? Wie kann eine lebendige Community aufgebaut und aufrechterhalten werden? Wie kann die Energie Einzelner, strategisch gebündelt und in Strukturen verortet werden? Wie können Kommunikationsformate genutzt werden, um den Veränderungsprozess zu unterstützen? Welche Bedeutung können Lehrpreise einnehmen?

Prof. Dr. Niclas Schaper

niclas.schaper@upb.de

1 Institut für Humanwissenschaften - Fach Psychologie, Universität Paderborn, Paderborn, Deutschland
Wie können diese Veränderungen evaluiert werden? In einem umfangreichen Rahmungskapitel werden auf diese Fragen Antworten gegeben, die zum Weiterdenken für eigene Veränderungsprozesse im Transformationsprozess genutzt werden können. Darüber hinaus wird beschrieben, wie man von der schonungslosen Reflektion des konsequent umgesetzten Veränderungsprozesses durch zentrale Akteure im Prozess profitieren kann.

Die beiden Herausgebenden, Simone Kauffeld, Inhaberin des Lehrstuhls für Arbeits-, Organisations- und Sozialpsychologie der TU Braunschweig und bis 2018 als Vizepräsidentin zuständig für den Aufbau der Innovativen Lehre und der Medienbildung sowie Julius Othmer, Verantwortlicher für die Projektgruppen in Studium und Lehre an der TU Braunschweig, haben gemeinsam mit weiteren zentralen Akteur/-innen ihren Weg an der TU Braunschweig, fachkundig beschrieben. Darüber hinaus haben sie in diesem Buch 34 praxisrelevante und dennoch theoretisch fundierte Beiträge zusammengebracht, rund um die Themen forschendes Lernen, mobiles Lernen, problemorientiertes Lernen, Game-based Learning, Inverted Classroom und Visualisierung.

Die Begeisterung der Autor/-innen für diese Methoden und Möglichkeiten neuer Lehr-Lern-Konzepte, spiegelt sich in diesem Buch. Die renommierten sowie durch Preise und Fördermittel ausgezeichneten Lehrenden stammen aus unterschiedlichen Fachrichtungen sowie verschiedenen Universitäten und Fachhochschulen in Deutschland. Neben den Praxisbeispielen von der TU Braunschweig, beschreibt z. B. Malte Persike von der Universität Mainz seine Erfahrungen und Erkenntnisse bei der Einführung des „Blended-Learning-Ansatzes" in Großveranstaltungen und thematisiert dabei nicht nur die Bedeutung digitaler Lernformate, sondern vor allem den Umgang mit den Studierenden in diesem Kontext (Thema: Inverted Classroom). 
Birgit Spinath, Professorin für Pädagogische Psychologie an der Universität Heidelberg, teilt ihre Erfahrungen eines Interviewprojekts zwischen Studierenden und Expert/-innen, welches in Kooperation mit dem Springerverlag anschließend veröffentlich wurde (,Meet the Expert: Wissen aus erster Hand"). Hier stand vor allem das selbstständige wissenschaftliche Arbeiten der Studierenden im Mittelpunkt (Thema: Forschendes Lernen).

Als weiteres, ganz anders ausgerichtetes Projekt, lässt sich das „Mytrack“ von Sönke Knutzen, Henning Klaffke und Alexander Schmitt der TU Hamburg Harburg herausstellen. Gemeinsam mit Studierenden wurde ein Online Tool entwickelt, welches sowohl bei der Diagnose von Kompetenzförderbedarfen als auch bei der Beratung von Studienanfänger/-innen unterstützen soll und so zu der Gestaltung eines individuellen Lernpfades dieser Zielgruppe führt (Thema: problemorientiertes Lernen).

Unter dem Themenpunkt „Mobiles Lernen“ stellen unter anderem Ulrich Foster und Friederike Hoffmann das Smartphone als Lernassistenten vor. In dem an der Universität Hohenheim durchgeführten Projekt wurde eine Software selbst entwickelt und im Einsatz erprobt. Ergebnis sind neue Chancen für realitätsnahe Lehre außerhalb der klassischen Lernräume.

Dem „Game-based Learning“ widmete sich z.B. Sandra Buron und berichtet in ihrem Beitrag über die Erfahrungen von "SimMed“, einer Simulation medizinischer Handlungen auf einem Multitouch-Tisch. Medizin Studierende durchleben simulierte virtuelle Untersuchungs- und Behandlungsszenarien, welche anschließend evaluiert und modifiziert werden können. Neben fachlicher Expertise fördert dieses Projekt die Kompetenz der Zusammenarbeit bei Studierenden und stellt somit einen wertvollen Beitrag für die Lehr-Lern-Möglichkeiten dar.

Einen einmaligen Vorgang in der bundesdeutschen Universitätsgeschichte beschreiben Andreas Jerrentrop, Tobias Müller, Andreas Neubauer und Jürgen Schäfer in einem Beitrag zum Themengebiet „Visualisierung“. Die US-amerikanische Serie „Dr. House“ inspirierte die Autoren zu der Neugestaltung einer Lehrveranstaltung zum Thema ,seltene Krankheiten“, welche besondere Aufmerksamkeit nicht nur bei Studierenden, sondern auch bei den Medien erregte und auf diese Weise zur Gründung eines Zentrums für unerkannte und seltene Erkrankungen an der Universitätsklinik Marburg führte.

Dies illustriert nur ansatzweise die Vielfalt der Beitrage innerhalb der breitgefächerten Themenschwerpunkte und zeigt die Relevanz dieses Buches für eine diverse Zielgruppe, welche die gleiche Vision verfolgt, Lehre zeitgemäß zu gestalten. Dabei werden Beispiel aus verschiedensten Fächer aufgezeigt, die allesamt das Potential haben auch auf andere Fächer übertragen zu werden.
Alle Thementeile sind nach einem einheitlichen Aufbau strukturiert und durch zusätzliche Querverweise übersichtlich organisiert. Dies erlaubt eine strukturierte Auseinandersetzung mit der Thematik, welche sowohl für Expert/-innen als auch Neueinsteigende geeignet ist.

Trotz der hohen Praxisrelevanz des Buches werden auch die theoretischen Fundierungen der vorgestellten Ansätze erläutert, Umsetzungsmöglichkeiten aufgezeigt und reflektierte Erfahrungsberichte mit dem Leser geteilt. Dies bietet die Möglichkeit, die beschriebenen Best-Practice-Ansätze als Anregung zum Transfer in die eigene Lehre zu nutzen. Dieses Buch leitet an, motiviert und inspiriert Lehre sowie die eigene Rolle neu zu interpretieren und die Möglichkeiten der heutigen Digitalisierung zu nutzen.

Das Buch bietet damit eine inspirierende Quelle für alle Lehrenden in der Hochschullehre und hochschuldidaktischen Weiterbildung. Darüber hinaus finden Trainer/-innen und Organisationsberater/-innen Anregungen für die berufliche Praxis. Um vom Experimentieren in die Breitenwirksamkeit zu gelangen, wie es im Geleitwort des Hochschulforums Digitalisierung formuliert ist, braucht es kritisch reflektierte Best Practice Beispiele, wie sie in diesem Buch von der Ebene einzelner Lehrveranstaltungen bis hin zu organisationalen Veränderungsprozessen dargestellt werden.

Funding Open Access funding provided by Projekt DEAL.

Open Access Dieser Artikel wird unter der Creative Commons Namensnennung 4.0 International Lizenz veröffentlicht, welche die Nutzung, Vervielfältigung, Bearbeitung, Verbreitung und Wiedergabe in jeglichem Medium und Format erlaubt, sofern Sie den/die ursprünglichen Autor(en) und die Quelle ordnungsgemäß nennen, einen Link zur Creative Commons Lizenz beifügen und angeben, ob Änderungen vorgenommen wurden.

Die in diesem Artikel enthaltenen Bilder und sonstiges Drittmaterial unterliegen ebenfalls der genannten Creative Commons Lizenz, sofern sich aus der Abbildungslegende nichts anderes ergibt. Sofern das betreffende Material nicht unter der genannten Creative Commons Lizenz steht und die betreffende Handlung nicht nach gesetzlichen Vorschriften erlaubt ist, ist für die oben aufgeführten Weiterverwendungen des Materials die Einwilligung des jeweiligen Rechteinhabers einzuholen.

Weitere Details zur Lizenz entnehmen Sie bitte der Lizenzinformation auf http://creativecommons.org/licenses/by/4.0/deed.de. 


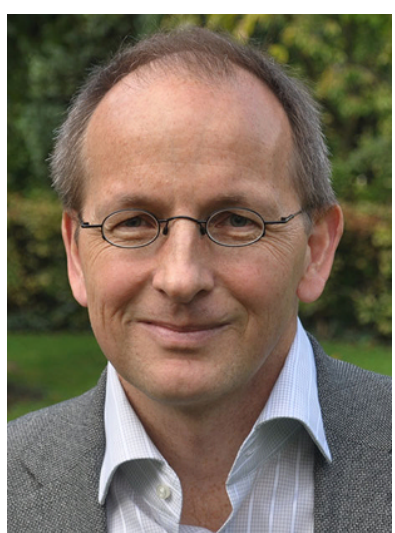

Prof. Dr. Niclas Schaper Universitätsprofessor und Hochschullehrer für Arbeits- und Organisationspsychologie sowie wissenschaftlicher Leiter der Stabsstelle für Bildungsinnovationen und Hochschuldidaktik an der Universität Paderborn. 